\title{
Identificación de sulfuros de arsénico complejos contenidos en una muestra mineral de Fresnillo, Zacatecas y propuesta de disolución de semimetales
}

\author{
A. M. Teja-Ruiz ${ }^{1}$, J.C. Juárez-Tapia ${ }^{1 *}$, K. Flores-Castro1, J. I. Martínez-Soto ${ }^{1}$, \\ M. Pérez-Labra ${ }^{1}$, J.E. Cruz-Peláez ${ }^{1}$, V. E. Reyes-Cruz ${ }^{1}$, M. Reyes-Pérez ${ }^{1}$ \\ ${ }^{1}$ Área Academica de Ciencias de la Tierra y Materiales, Universidad Autónoma del Estado de Hidalgo, \\ Carretera Pachuca-Tulancingo Km 4.5, Mineral de la Reforma, Hidalgo, 42180, MÉXICO). \\ aislinn_teja@uaeh.edu.mx,jcjuarez@uaeh.edu.mx,kinardo_flores@hotmail.com, ivan_kai@outlook.es, \\ miguelabra@hotmail.com,emmanuel_cruz97@hotmail.com, reyescruz16@yahoo.com, \\ mreyes@uaeh.edu.mx
}

*Autor de correspondencia: jcjuarez@uaeh.edu.mx

\section{RESUMEN}

En la industria minera, los concentrados de cobre, plomo y zinc son comúnmente castigados de manera comercial por su alto contenido de arsénico. En busca de combatir esta problemática, se caracterizó un mineral proveniente de Fresnillo, Zacatecas, con importantes concentraciones de este metal tóxico a fin de someterlo a un baño alcalino y lograr la separación del arsénico del resto del mineral. El análisis químico realizado a la muestra del mineral mediante la técnica de Espectroscopia de Emisión de Plasma Acoplada por Inducción (ICP) y Fluorescencia de Rayos X (FRX) identificó un alto contenido de $\mathrm{Fe}(17.94 \% \mathrm{w} / \mathrm{w})$ y As $(12.95 \% \mathrm{w} / \mathrm{w})$, los cuales forman parte del sulfuro complejo identificado como arsenopirita (FeAsS) [96-900-0110]. Además, la presencia de estos elementos fue corroborada mediante las técnicas de DRX, MEB-EDS y MOP. Estas ténicas tambien revelaron la presencia de sulfuros de metales base como ZnS y PbS, así como especies de ganga como $\mathrm{SiO}_{2}$ y algunos elementos minoritarios como: $\mathrm{Sb}, \mathrm{Ag}, \mathrm{Cu}$, $\mathrm{Zn}$ y $\mathrm{Pb}$. La construcción de diagramas de Pourbaix utilizando el sistema Fe-As-S- $\mathrm{NaOH}$ $\mathrm{H} 2 \mathrm{O}$ a $25^{\circ} \mathrm{C}$, permitió determinar que las especies $\mathrm{AsO}_{4}{ }^{3-}$ y $\mathrm{FeO}{ }^{*} \mathrm{OH}$ predominan bajo las condiciones experimentales a las que se llevaron a cabo las pruebas preliminares ([Mineral] $=8 \mathrm{~g} \mathrm{~L}^{-1},[\mathrm{NaOH}]=2 \mathrm{~mol} \mathrm{~L}^{-1}, 25{ }^{\circ} \mathrm{C}, \mathrm{RPM}=800 \mathrm{~min}^{-1}$ y $720 \mathrm{~min}$ ), logrando la disolución de $47 \%$ del semimetal.

Palabras Clave: Arsénico, Sulfuro Complejo, Semimetal, Caracterización, Disolución.

\section{INTRODUCCIÓN}

El arsénico se encuentra relacionado de forma natural con especies minerales cuyo contenido elemental representa una importante fuente de extracción de metales, como es el caso del oro, la plata, el plomo, el zinc, el hierro y el cobre [1]. En el caso de los metales nobles, la presencia del arsénico se refleja en la formación de sulfuros complejos y especies de carácter refractario que dificultan la lixiviación del metal precioso [2, 3]. Cuando se trata de concentrados de metales base la presencia de arsénico es considerada como un contaminante que suele ser castigado en la comercialización de los concentrados [4], lo que convierte a la presencia de este semimetal en un inevitable problema a tratar en los procesos hidrometalúrgicos. Por otra parte, en los países con 
una importante tradición minera como: Chile, Perú, Canadá y México, sus principales yacimientos se enfrentan a la escasez de minerales simples, lo que ha obligado al uso de operaciones mineras de mayor profundidad, donde las especies de arsénico son más abundantes [5]. Es por ello que la búsqueda de alternativas para la eliminación del arsénico ha tomado importancia en el campo de la industria de la metalurgia extractiva. Estudios anteriores han reportado la oxidación mecano-química de un mineral de arsenopirita a través del uso de peróxido de hidrogeno en un medio acuoso a $50^{\circ} \mathrm{C}$, sin embargo, únicamente se reportó la liberación del 9\% de hierro y 7\% de arsénico tras 120 minutos de reacción [6, 7]. En los concentrados multitemáticos obtenidos mediante el proceso de flotación es común detectar la presencia de arsénico relacionado con el mineral de arsenopirita [8, 9]. En trabajos previos se ha reportado los mecanismos de depresión del mineral de arsenopirita, al lograr la oxidación del fierro de las partículas de arsenopirita, logrando disminuir la flotabilidad de este mineral que contienen al semimetal [10]. Por otro lado, estudios relacionados con la biolixiviación de minerales de baja ley han reportado que la presencia de arsenopirita promovió un incremento en la población de los microorganismos y permitió la degradación de azufre elemental, sin embargo, el arsénico sigue presente en el sólido mineral al formar la especie de oropimente $\left(\mathrm{As}_{2} \mathrm{~S}_{3}\right)$ $[11,12]$. Estas alternativas representan, en su mayoría, la adición de una etapa previa al proceso de lixiviación tradicional la cual, aunque tiene la finalidad de transformar el mineral de alimentación para su eficiente extracción, también involucra un aumento en los costos de operación. El territorio minero de la Ciudad de Fresnillo en Zacatecas, esta principalmente caracterizada por especies mineralógicas conformadas por abundantes concentraciones metálicas como As, Au, Cu, Fe, Pb y Zn [13]. El presente trabajo tiene como objetivo identificar la especie mineral relacionada con la presencia de arsénico y generar una propuesta de disolución del semimetal mediante soluciones de $\mathrm{NaOH}$ altamente alcalinas cuyo escalamiento industrial resulte más rentable.

\section{PARTE EXPERIMENTAL}

La muestra de mineral fue obtenida en el distrito minero localizado en ciudad de Fresnillo, Zacatecas, México. La muestra se preparó mecánicamente hasta alcanzar un diámetro de partícula menor a 74 micras. Posteriormente fue sometida al método de cuarteo, con la finalidad de obtener una muestra representativa de un $0.5 \mathrm{Kg}$, con la que se realizó la caracterización química y mineralógica del mineral. Para identificar las especies mayoritarias presentes en el mineral se utilizó la técnica de Difracción de rayos X (DRX) usando difractómetro de rayos X marca INEL EQUINOX 2000, con una radiación Cu-Ka1 de 1,540598, operando a $30 \mathrm{~mA}$ y $20 \mathrm{KV}, 220 \mathrm{~V}$ y una resolución de 0.095 FWHM.

El contenido elemental de la muestra fue analizado mediante la técnica de Fluorescencia de rayos $X(F R X)$, para lo cual se analizaron los polvos de la muestra utilizando un espectrómetro de fluorescencia de rayos $X$ marca Bruker modelo S2 PUMA con tubo de paladio. Para corroborar la información obtenida del contenido elemental se realizó la disolución acida de 1 gramo de mineral. La solución resultante fue filtrada, aforada a 0.1 L y analizada mediante la técnica de Espectroscopia de Emisión de Plasma Acoplada por Inducción (ICP) usando un espectrómetro Perkin Elmer 8300. Para la identificación de las especies minerales presentes en la muestra mineral, los polvos de la misma fueron embebidos en resina epóxica para obtener una probeta. La cual fue pulida en acabado espejo y analizada mediante las técnicas de Microscopia Electrónica de Barrido- Análisis 
de dispersión de Energías (MEB-EDS), usando un microscopio electrónico de barrido marca JEOL modelo JSM 6701F a 25 KV y Microscopia Óptica de Polarización (MOP) usando un microscopio óptico de polarización modelo $\mathrm{BH} 41$ con diferentes magnificaciones. Las pruebas preliminares se realizaron por triplicado con la finalidad de corroborar el comportamiento de la reacción de disolución.

Las condiciones experimentales utilizadas para la disolución de arsénico fueron: $V=0.25$ $\mathrm{L}$, mineral $=8 \mathrm{gL}^{-1}, \mathrm{t}_{\mathrm{r}}=720 \mathrm{~min}, \mathrm{~d}_{0}=-105+74 \mu \mathrm{m},[\mathrm{NaOH}]=3 \mathrm{~mol} \mathrm{~L}^{-1}, \mathrm{~T}=298 \mathrm{~K}$ y RPM $=1000 \mathrm{~min}^{-1}$, y se usó un reactor de vidrio con capacidad de $500 \mathrm{~mL}$ sobre una parrilla de calentamiento marca ThermoScientific Super Nuova equipada con agitación magnética. A partir de las pruebas preliminares se realizó la medición del pH y Eh usando un pHmetro marca STARA2115 OrionThermoScientific, con la finalidad de identificar las especies predominantes en los diagramas de Pourbaix construidos con base al sistema Fe-As-S- $\mathrm{H}_{2} \mathrm{O}$ a una temperatura de $298 \mathrm{~K}$ utilizando el HSC Chemistry software 5.11 (Outotec, Cleveland, OH, USA).

\section{RESULTADOS}

A continuación, se presenta la caracterización química y mineralógica realizada a partir de una muestra mineral proveniente del municipio de Fresnillo en el estado de Zacatecas.

\section{Difracción de Rayos X (DRX)}

Mediante la técnica de DRX se logró identificar al cuarzo ( $\left.\mathrm{SiO}_{2}\right)$ como la matriz mineral de la muestra, una especie considerada como un componente ganga. Sin embargo, el contenido metálico de mayor importancia se encuentra relacionado con especies de hierro, identificadas como arsenopirita, un sulfuro de arsénico con hierro, y goethita, oxihidróxido de hierro. Ambas especies se asocian con filones hidrotermales, en presencia de vetas de cuarzo y oro [14].

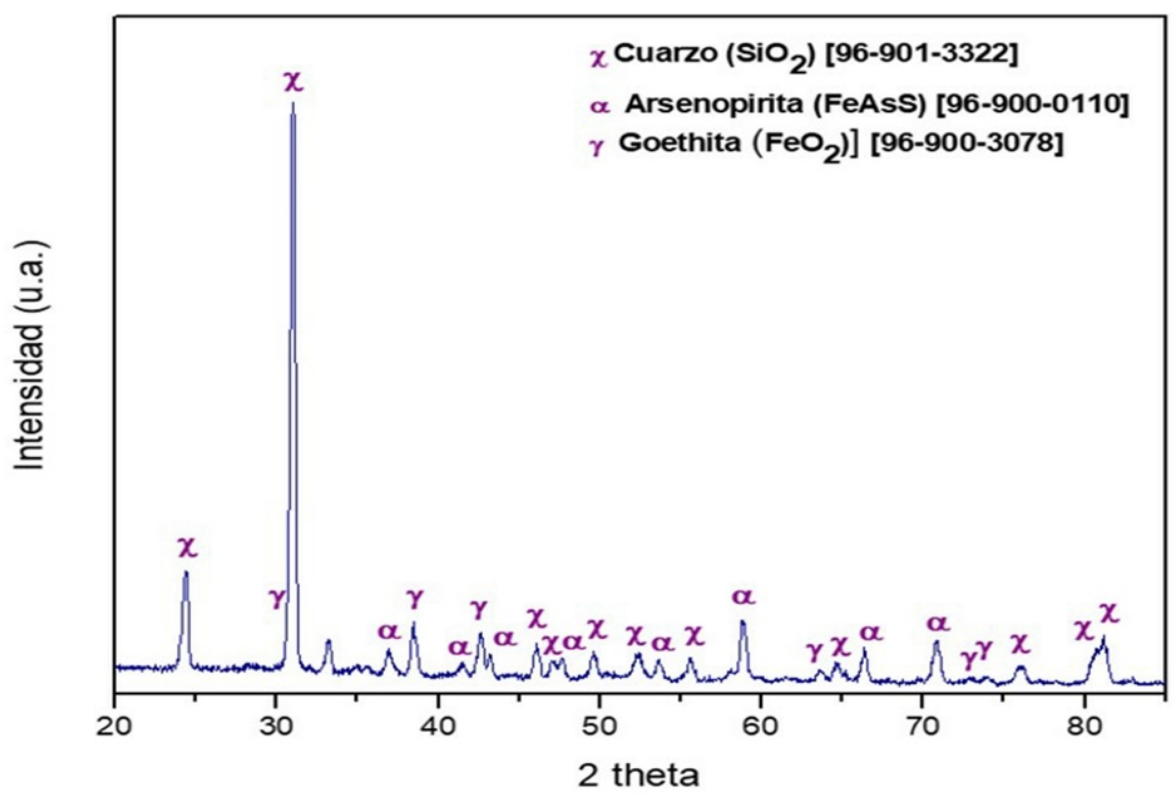

Figura 1. Difractograma de la muestra mineral de Fresnillo, Zacatecas 


\section{Espectroscopia de Emisión de Plasma Acoplada por Inducción (ICP)}

El análisis químico realizado a la muestra mineral fue dirigido únicamente para conocer el contenido metálico de la muestra y los elementos de interés económico, entre los que destaca principalmente la concentración de Fe $(17.94 \% \mathrm{w} / \mathrm{w})$, seguido del arsénico $(12.95 \% \mathrm{w} / \mathrm{w})$. El resto de metales de menor abundancia son elementos relacionados principalmente con la presencia de sulfuros base como el $\mathrm{Zn}, \mathrm{Pb}$ y $\mathrm{Cu}$. Los elementos más escasos en la muestra, aunque de mayor interés económico son la plata y el antimonio, elementos comúnmente asociados a la formación de sulfosales complejas.

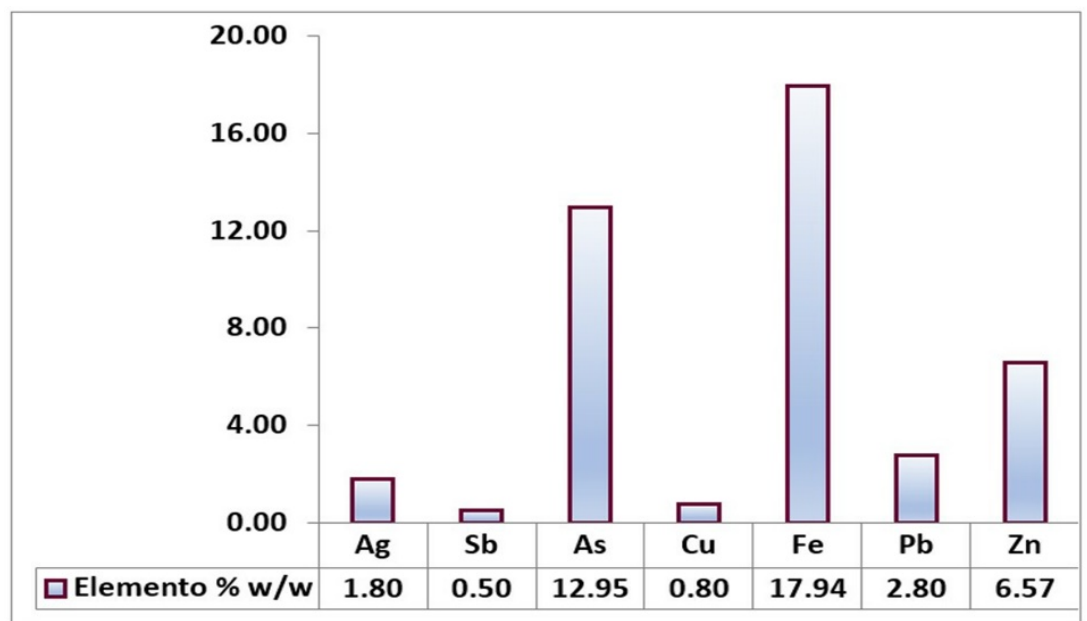

Figura 2. Análisis químico realizado a la muestra mineral de Fresnillo, Zacatecas obtenido mediante ICP.

\section{Fluorescencia de Rayos X (FRX)}

Con la finalidad de corroborar el contenido elemental de la muestra se analizaron los polvos de la misma por triplicado, utilizando la técnica FRX, donde además del contenido metálico se identificó la presencia de elementos traza. Con base a las especies mineralógicas identificadas mediante DRX, se logró relacionar los elementos de mayor abundancia, como es el caso del Si y O, elementos que conforman la matriz del mineral [15]. Por otra parte, se observó importantes concentraciones de Fe, As y S, sin embargo, el contenido de azufre duplica el contenido del Fe y el As, esto se atribuyó a la presencia se sulfuros de metales base característicos del tipo de yacimiento.

Tabla 1. Contenido elemental (\% w/w) de la muestra mineral de Fresnillo, Zacatecas mediante

\begin{tabular}{|c|c|c|c|c|c|c|c|c|c|c|c|c|}
\hline Elemento & Ag & Sb & Pb & As & Zn & Cu & Fe & Ca & K & S & Si & O \\
\hline $\begin{array}{c}\text { Promedio } \\
\text { (\% w/w) }\end{array}$ & 1.75 & 0.51 & 2.57 & 12.51 & 7.63 & 0.08 & 15.63 & 0.66 & 0.32 & 22.09 & 12.96 & 23.30 \\
\hline
\end{tabular}

\section{Microscopia Óptica de Polarización (MOP)}

Mediante la técnica de MOP, se obtuvieron una seria de micrografías mediante las cuales se identificaron las especies metálicas con base a sus características ópticas. En la figura 
3a, se observa la presencia de pirita cuyos ángulos rectos y tonalidad brillante corroboran su hábito cristalino característico, la presencia de arsenopirita se confirmó mediante la identificación de ángulos agudos característicos de su estructura triclínica, al igual que en la figura 3b, donde se observa un partícula desbastada de arsenopirita, cuya tonalidad clara contrasta con la partícula oscura que se observa en la parte inferior de la partícula, la cual corresponde a la roca encajonante [16].

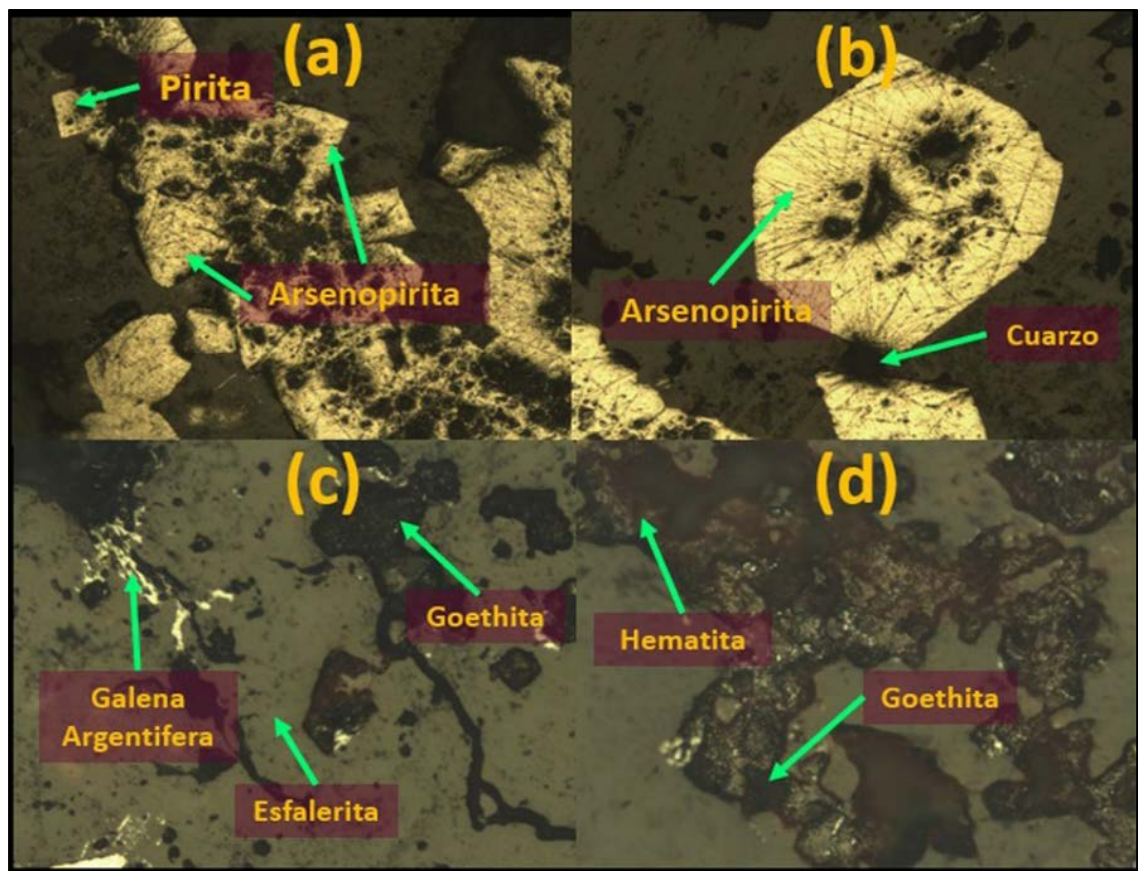

Figura 3. Micrografía de la muestra mineral de Fresnillo Zacatecas obtenida mediante nicoles polarizados a 10x en presencia de: (a) Pirita y arsenopirita (b) Arsenopirita y cuarzo (c) Galena argentífera, esfalerita y goethita (d) Hematita y goethita.

En la micrografía 3c y 3d, se observó la presencia de óxidos de hierro, correspondientes a Hematita (tonalidades rojizas) y la Goethita (tonalidades oscuras), esta última siendo una especie mayoritaria identificada mediante DRX. En la micrografía 3c, se observó la presencia de sulfuros de metales base, como esfalerita y galena argentífera, lo que confirma los porcentajes en peso obtenidos a partir del análisis químico elemental, así como la relación del sulfuro de plomo con el contenido de plata identificado mediante ICP y FRX.

Microscopia electrónica de barrido- Análisis de energías dispersivas (MEB-EDS). Mediante el uso de electrones retrodispersados en la técnica de MEB-EDS, se obtuvo la micrografía de una partícula metálica cuya relación elemental entre $\mathrm{S}$, Fe y As, determinada por la técnica de mapeo, permitió identificarla como la especie arsenopirita, como lo muestra la figura 4.

El barrido realizado para los elementos $\mathrm{C}, \mathrm{O}, \mathrm{Si}, \mathrm{S}, \mathrm{Fe}, \mathrm{As}$ y $\mathrm{Zn}$, permitieron corroborar la existencia de esfalerita en la muestra mineral y a su vez identificar partículas de tonalidades oscuras correspondientes al cuarzo [14]. El contenido de $\mathrm{C}$ y $\mathrm{O}$, corresponden a la resina epóxica, utilizada en la preparación de la muestra. El análisis 
semicuantitativo realizado a la partícula metálica confirmo la composición del sulfuro de arsénico con hierro.

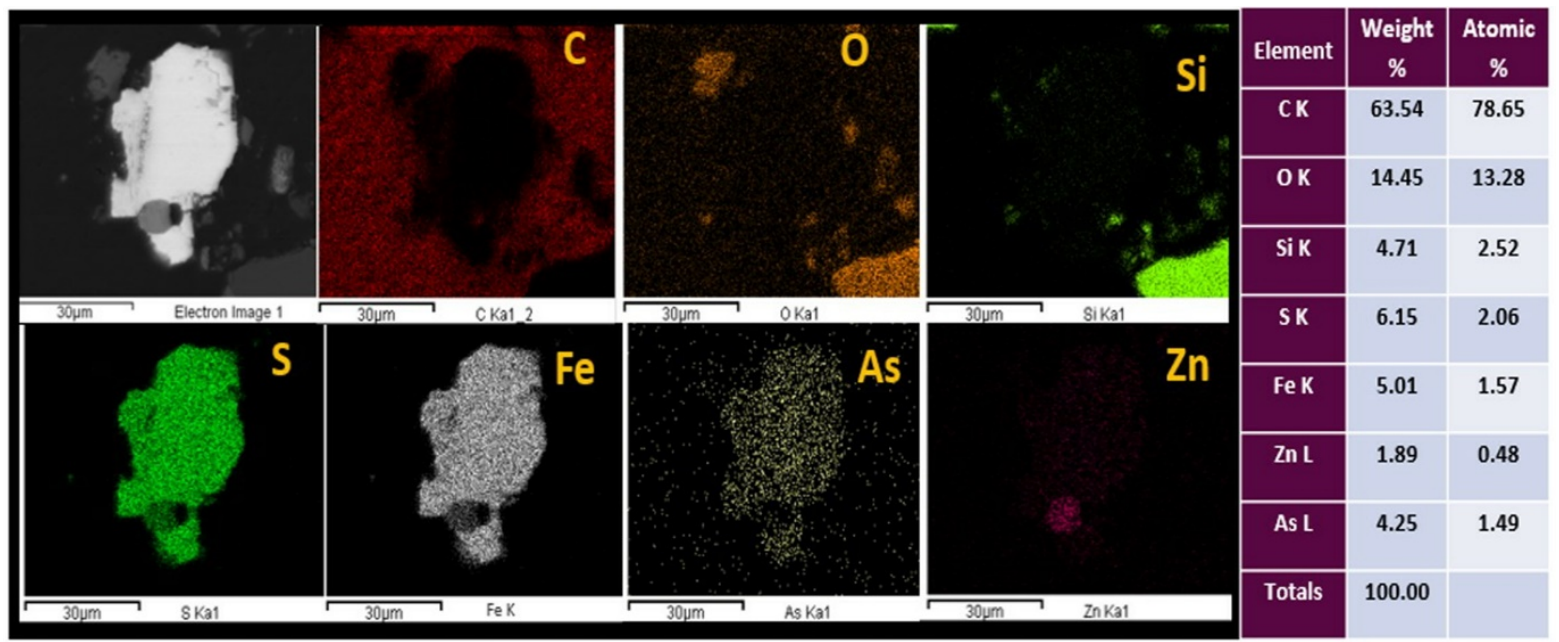

Figura 4. Mapeo realizado a una partícula metálica de la muestra mineral de Fresnillo, Zacatecas y análisis semicuantitativo.

\section{Estudio de especiación}

Los diagramas de Pourbaix obtenidos para el sistema As-Fe-S-NaOH-H $2 \mathrm{O}$ a $25{ }^{\circ} \mathrm{C}$ establecieron las condiciones teóricas para la obtención del ion $\mathrm{AsO}_{4}{ }^{3-}$ ante la presencia de iones de sodio libres en un amplio rango de $\mathrm{pH}$ y a potenciales oxidantes (figura $5 \mathrm{a}$ y 5b).

El monitoreo de las pruebas de disolución permitió corroborar las especies predominantes de As y Fe al alcanzar un $\mathrm{pH}$ equivalente a 12.03 y un potencial de 0.2 volts. Bajo dichas condiciones se establecieron las especies de dominio que conforman el sistema propuesto y se construyó la siguiente relación estequiométrica que da lugar a la reacción de disolución de As (ecuación 1):

$$
\mathrm{FeAsS}+2 \mathrm{NaOH}+8 \mathrm{H}_{2} \mathrm{O} \rightarrow \mathrm{AsO}_{4}^{3-}+\mathrm{FeO} * \mathrm{OH}+\mathrm{SO}_{4}^{2-}+2 \mathrm{Na}^{1+}+17 \mathrm{H}^{+}
$$

Como se observa la ecuación 1, el baño alcalino con $\mathrm{NaOH}$, permite la conversión tanto del Fe como del As a especies oxidadas (figura 6c). Mientras que el contenido de azufre correspondiente a la especie arsenopirita reacciona en el medio formando iones sulfato a $\mathrm{pH}$ altamente alcalino. 

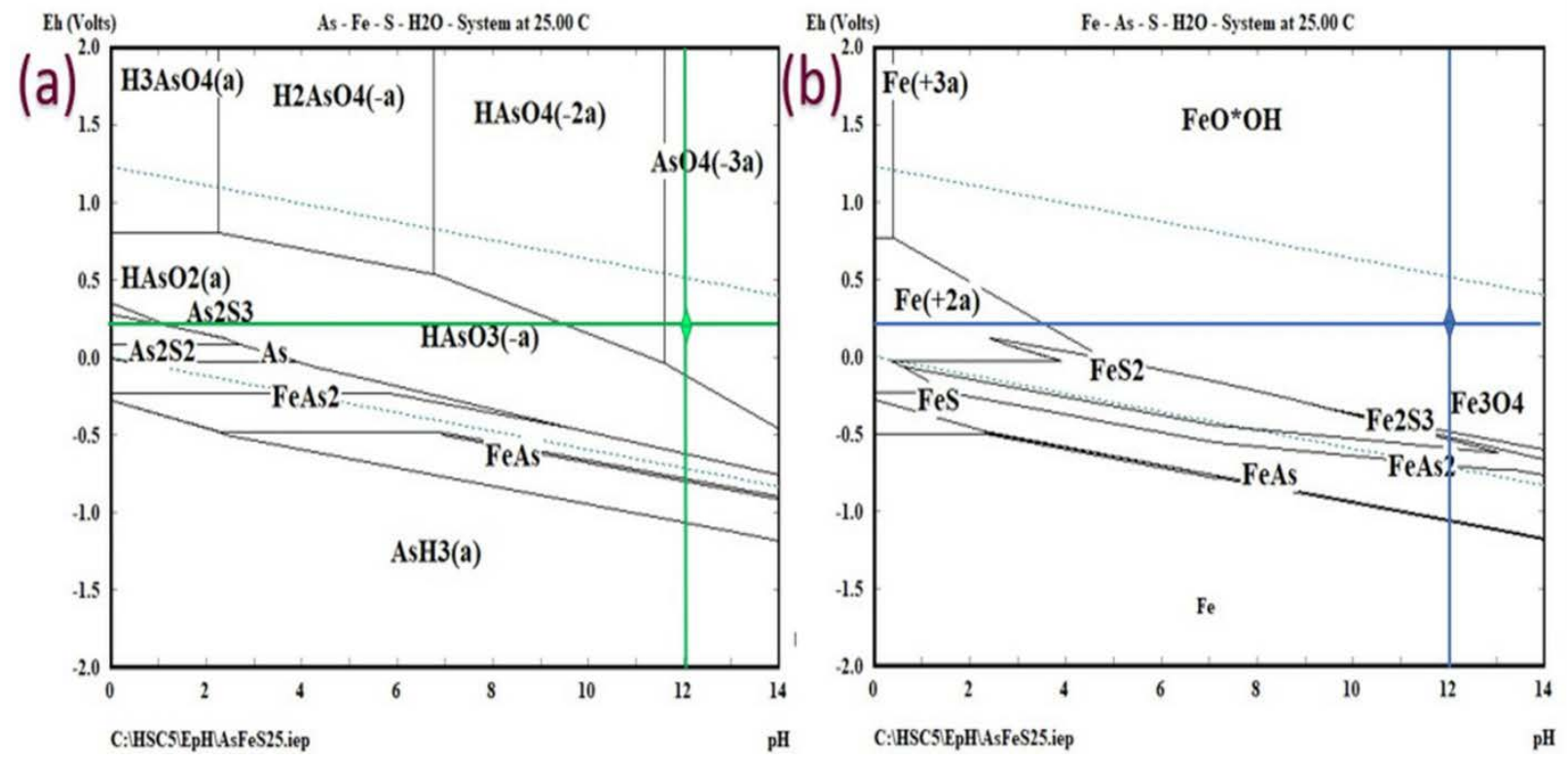

Figura 5. Diagramas de Pourbaix correspondientes al sistema As-Fe-S-NaOH-H $\mathrm{H}_{2} \mathrm{O}$ a $25{ }^{\circ} \mathrm{C}$ a $[\mathrm{As}]=0.12 \mathrm{~m} \mathrm{~L}^{-1},[\mathrm{Fe}]=0.15 \mathrm{~m} \mathrm{~L}^{-1}$ y $[\mathrm{S}]=0.22 \mathrm{~m} \mathrm{~L}^{-1}$. (a) Especies dominantes de As (b) Especies dominantes de Fe.

\section{Eliminación de arsénico mediante el uso soluciones concentradas de $\mathrm{NaOH}$}

Las pruebas preliminares para la disolución de arsénico a partir de la especie arsenopirita, se realizaron por triplicado con la finalidad de corroborar el comportamiento de la reacción. La disolución de As en solución que se presenta en la figura 6, confirma la eliminación del $47 \%$ del semimetal contenido en la especie arsenopirita a partir del baño alcalino propuesto.

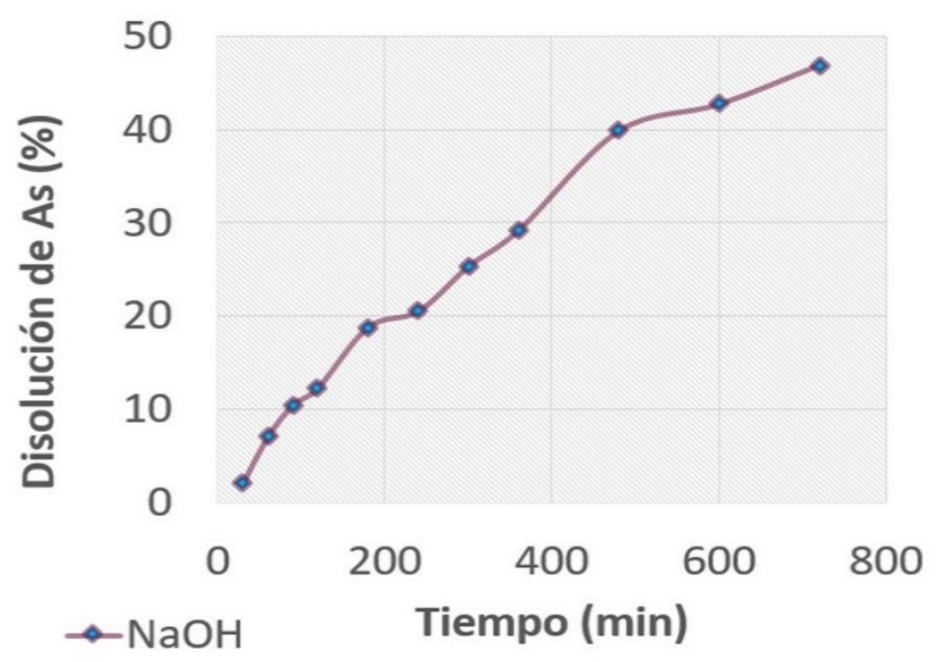

Figura 5. Curva de disolución de arsénico en el sistema alcalino FeAsS- $\mathrm{NaOH}-\mathrm{H}_{2} \mathrm{O}$. 


\section{CONCLUSIONES}

La caracterización mineralógica permitió identificar al cuarzo como la matriz de la muestra mineral, sin embargo, la especie metálica de mayor interés económico es la arsenopirita, un sulfuro de Fe y As, cuyo contenido elemental fue corroborado a su vez por la técnica de ICP y FRX. La presencia de otros sulfuros de metales base como la galena, la pirita y la esfalerita también fueron identificadas en menor abundancia mediante MOP, MEBEDS y DRX.

La estequiometria propuesta para el sistema de disolución de As a partir de la especie arsenopirita mediante el uso de soluciones altamente alcalinas se fundamentó mediante la construcción de diagramas de Pourbaix, los cuales permitieron identificas los rangos de Eh y $\mathrm{pH}$ a los que se obtienen las especies predominantes de Fe y As. Mediante las pruebas preliminares se alcanzó un $\mathrm{pH}=12.03$ y un potencial de 0.2 volts, confirmando la conversión del arsénico a especies iónicas y alcanzando disoluciones máximas del semimetal correspondientes a un $47 \%$.

\section{BIBLIOGRAFÍA}

[1]. Ballester, L. Verdeja, J. Sancho. Metalurgia Extractiva Vol. II, 2nd ed., Síntesis, Madrid, España (2013) 42-49.

[2]. I. Alp, O. Celep, D. Paktunç, Y. Thibault. Hydrometallurgy. 146 (2014) 64-71.

[3]. A.R. Alonso-Gómez, G. T. Lapidus. Hydrometallurgy. 99 (2009) 89-96.

[4]. A. M. Teja-Ruiz, J. C. Juárez-Tapia, I. A. Reyes-Domínguez, L. E. Hernández-Cruz, M. Reyes-Pérez, F. Patiño-Cardona, M. U. Flores-Guerrero. Metals MDPI, 7 (2017) 1-16.

[5]. E. González-Partida, A. Carrillo-Chávez, G. Levresse, J. Tritlla, A. Camprub. Ore Geol. Rev. 23 (2003) 91-96.

[6]. L. Koroznikova, S. McKnight, J. P. Veder, J. Giri, S. Palaniandy, G. Williams. Minerals Engineering. 83 (2019) 24-36.

[7]. J. Hong, L. Liu, Y. Luo, W. Tan, G. Qiu, F. Liu. Geochimica et Cosmochimica Acta. 239 (2018) 173-185.

[8]. J. Ran, X. Qiu, Z. Hu, Q. Liu, B. Song, Y. Yao. Applied Surface Science. 480, (2019) 1136-1146.

[9]. J. Lu, Z. Tong, Z. Yuan, L. Li. Minerals Engineering. 137 (2019)118-123.

[10]. L. Yu, Q. Liu, S. Li, J. Deng, B. Luo, H. Lai. Separation and Purification Technology. 222(2019) 109-116.

[11]. Y. K. Kawa, J. Wang, X. Chen, X. Zhu, X.C. Zeng, Y. Wang. International Biodeterioration \& Biodegradation. 143 (2019) $104-112$.

[12]. D. R. Zhang, J. L. Xia, Z. Y. Nie, H. R. Chen, H. C. Liu, Y. Deng, Y. D. Zhao, L. Z. Zhang, W. Wen, H. Y. Yang. Process Biochemistry. 81 (2019) 11-21.

[13]. Servicio Geológico Mexicano (SGM). Edición de cartas Geológico-mineras y $\begin{array}{llll}\text { geoquímicas } & \text { escala } & 1: 50,000 . & 018.07-2019 .\end{array}$ http://www.sgm.gob.mx/cartas/Cartas_Ed50.jsp 
[14]. W. A. Deer, R. A. Howie, J. Zussman. An introduction to the rock-forming Minerals, $2^{\text {nd }}$ ed., Longman, Hong Kong, China (1998) 584-611.

[15]. T. C. Robson, C.B. Braungardt, M. J.Keith-Roach, J. S. Rieuwerts, P. J. Worsfold. Journal of Geochemical Exploration. 125 (2013) 102-109.

[16]. A. Murciego, E. Álvarez-Ayuso, S.C. Aldana-Martínez, A. Sanz-Arranz, J. MedinaGarcía, F. Rull-Pérez, P. Villar-Alonso. Journal of Hazardous Materials. 373 (2019) 425-436 